\section{GP201 INCIDENCE AND CLINICAL PRESENTATION OF SEROLOGICALLY CONFIRMED PAEDIATRIC LYME DISEASE IN IRELAND OVER A 5 YEAR PERIOD}

${ }^{1}$ Karina Forde*, ${ }^{2}$ Bartley Cryan, ${ }^{3}$ Deirbhile Keady, ${ }^{3}$ Belinda Hanahoe, ${ }^{4}$ Lorraine Power, ${ }^{5} \mathrm{Colm}$ McDonnell, ${ }^{6,7}$ Patrick Gavin, ${ }^{8}$ Matthew Dryden, ${ }^{9}$ Joanne O'Gorman ${ }^{1}$ Michael O'Grady. 'Department of Paediatrics, Midland Regional Hospital, Mullingar, Mullingar, Ireland; 'Department of Microbiology, Cork University Hospital, Cork, Ireland; ${ }^{3}$ Department of Microbiology, University Hospital Galway, Galway, Ireland; ${ }^{4}$ Department of Microbiology, University Hospital Limerick, Limerick, Ireland; ${ }^{5}$ Department of Microbiology, University Hospital Limercik, Limerick, Ireland; ${ }^{6}$ Paediatric Infectious Diseases, Temple Street Children's University Hospital, Dublin, Ireland; 'Paediatric Infectious Diseases, Our Lady's Children's Hospital Crumlin, Dublin, Ireland; ${ }^{8}$ Rare and Imported Pathogens Laboratory, Public Health England, Porton Down, UK; ${ }^{9}$ National Viral Reference Lab, Dublin, Ireland

10.1136/archdischild-2019-epa.261

Background and aims Despite the fact that one of the peaks of Lyme disease incidence occurs in childhood, there are no population-based studies of incidence in children in Ireland, or indeed Europe. We aimed to identify the incidence and clinical presentation of serologically confirmed Lyme disease in patients aged 1-18 years in Ireland over a 5-year period. Methods A cross-sectional survey was conducted across all four laboratories in Ireland who perform in-house ELISA testing for Borrelia species (accredited to ISO 150189 standard). Between 2012-2016, all paediatric samples that were ELISA positive underwent confirmatory Western Blot testing through the Lyme Reference laboratory in the UK. For patients who were two-tier positive, an anonymous proforma was distributed by the respective Irish laboratories to their requesting clinicians to collect clinical details regarding their presentation, treatment and outcome.

Results 64 patients aged 1-18 with two-tier positive Borreliaserology were identified, representing just $2 \%$ of 2914 samples tested (1.1 per 100,000 children aged $1-18$ per year). Proformas were returned for 52 (87\%), of whom 48 (92\%) had a clinical presentation consistent with Lyme disease. The mean age at presentation was 9.5 years. 27 (51.9\%) cases were reportedly contracted in Ireland, predominantly in the west, and 22 children $(45.8 \%)$ recalled a tick bite.

$27(56 \%)$ children in our cohort were characterised as having Lyme Disease (LD) without focal symptoms. 19 (70\%) of those had solitary erythema migrans, and 3 (11.1\%) had multiple erythema migrans. $92.5 \%$ of those with LD without focal symptoms were treated with oral antibiotics. Full symptom resolution was documented in $88.8 \%$ of cases.

$20(41.6 \%)$ children were characterised as having LD with focal symptoms. 11 (22.9\%) had cranial nerve palsy without associated CNS involvement, and one child (2\%) had arthritis. 8 children (16.6\%) had LD with central nervous system involvement. Of the 19 children with CNS or cranial nerve involvement, $7(36.8 \%)$ had a history of erythema migrans, involving the head/neck in all cases. Full symptom resolution was documented in $95 \%$ of children with LD with focal symptoms.

There were no cases of carditis. No patient had a post Lyme disease syndrome. Of the 44 children in our cohort with documented antimicrobial treatment, treatment duration was appropriate in 39 cases (88.6\%).

Conclusions Despite increasing public awareness, Lyme disease remains rare in Irish children. Presentation was predominantly with erythema migrans and neurological manifestations and importantly, all children for whom data were available, recovered with no long-term sequelae

\section{GP203 PAEDIATRIC NOSOCOMIAL AND HEALTH CARE WORKER TRANSMISSION DURING MEASLES OUTBREAK, DUBLIN 2018}

Louise Marron*, Ruth McDermott, Robert Conway, Orla Ennis, Orla Bruton, Mary Ward Helena Murray. Department of Public Health East, Dublin, Ireland

\subsection{6/archdischild-2019-epa.262}

Background Despite achieving measles elimination status in 2018, Ireland remains vulnerable to measles outbreaks following imported cases. Overall uptake of two doses of MMR vaccine is suboptimal. Pockets of under immunised populations vulnerable to measles reflect anti-vaccination sentiment as well as difficulty accessing services among deprived and marginalised communities. A measles outbreak occurred in North Dublin in July 2018 with important public health and health service implications including nosocomial paediatric transmission and health care worker transmission in an adult hospital.

Methods A delayed diagnosis of measles in a child recently returned from Europe was the index case in a measles outbreak which spread in healthcare settings and within an inner city area. Epidemiological investigation determined demographic characteristics, vaccination status and transmission pathways of cases. Management of the outbreak included isolation, contact tracing, exclusions, and post exposure prophylaxis with MMR vaccine and immunoglobulin for appropriate contacts. Public health alerted general practitioners, adult and paediatric emergency departments, microbiologists and occupational health physicians to the outbreak. Social inclusion and community health services supported targeted control measures for vulnerable exposed communities. An extensive awareness campaign was undertaken involving print, radio, TV and geo-targeted social media messaging.

Results There were 17 confirmed cases. Eight (47.1\%) occurred in adults and nine $(52.9 \%)$ in children. Four $(23.5 \%)$ were under 12 months old. Two (11.8\%) were health care workers with complete MMR vaccination and reported relatively mild illness. Thirteen cases $(76.5 \%)$ attended emergency departments and four (23.5\%) required hospitalisation. Eleven (64.7\%) were unvaccinated, including four infants $(23.5 \%)$. The primary case was travel acquired; household transmission occurred in seven cases (41.2\%); nosocomial transmission occurred in six cases (35.3\%) and in three cases $(17.6 \%)$ the route of transmission was unknown. Over 400 children were exposed in emergency healthcare settings resulting in three attributable cases. Measles transmission also occurred between paediatric inpatients. Eight infants received human normal immunoglobulin following exposure.

Implications Following an imported case of measles, transmission occurred from multiple healthcare exposures and within under-vaccinated households in socially deprived areas. This outbreak highlighted: delayed recognition of measles facilitating nosocomial transmission; unusual clinical presentations among adults; vulnerable unvaccinated populations including children under 12 months and transmission to vaccinated healthcare workers which did 
not result in onward transmission. In addition recent changes in the national guidelines for providing post exposure prophylaxis with immunoglobulin to young infants presented logistical challenges when delivered in the hospital setting.

\section{GP204 HIV INFECTION IN CHILDREN WITH PERINATAL TRANSMISSION: CLINICAL AND IMMUNOLOGICAL FEATURES}

\begin{abstract}
'Ekaterina Moskaleva, 'Alla Petrova, ${ }^{2}$ Svetlana Smirova, 'Lyubov Rychkova, ${ }^{1}$ Anastasiya Vanyarkina*. 'Scientific Centre for Family Health and Human Reproduction Problems, Irkutsk, Russian Federation; ${ }^{2}$ Scientific Research Institute of Medical Problems of the North, Krasnoyarsk, Russian Federation
\end{abstract}

10.1136/archdischild-2019-epa.263

Introduction HIV infection is more rapid and serious in children with perinatal transmission. As the immune system is progressively deteriorating, the aim was to study parameters of the immune system, which is necessary to determine disease approach and prognosis.

Methods We conducted a cross-sectional randomized study of children aged 1 to 5 years: 32 children with perinatal HIV infection (main group), and 28 healthy children. Criteria for inclusion in main group: confirmed HIV infection, clinical stages 2-3 (WHO classification, 2006). Exclusion criterion is parents' refusal to participate. Clinical examination of children was conducted. We estimated the frequency of clinical manifestations of immunodeficiency syndromes $\left(\mathrm{P}_{\mathrm{abs}} / \mathrm{P} \%\right)$, and analyzed parameters of the immune status. The pattern of lymphocyte subpopulations was assessed by laser flow cytometry, immunoglobulins and interleukins concentrations were determined by EIA. A comparative analysis was performed using non-parametric Mann-Whitney U-test.

Results Regardless of clinical stage, all children with HIV infection showed delay in physical and neuropsychic development, CNS damage, Banti's syndrome, bulky lymphadenopathy, recurrent bacterial infections of upper respiratory tract. Frequent manifestations of HIV infection are anemia of 90.5 $\pm 3.7 \%$, and repeated pneumonia from 2 to 6 times a year $(71.4 \pm 5.7 \%)$. Immunity disorders manifested themselves in all HIV-infected children. Infectious and lymphoproliferative syndromes have the most intensive manifestation and are found in $100 \%$ of the HIV-infected. Infections target organs and systems, functioning as a barrier and participating in immunogenesis.

Combined damage to the immune system was detected. The state of cellular immunity was characterized by decreased numbers of CD3 + lymphocytes $(55.3 \pm 7.05 \%$; $\mathrm{p}<0.01)$, CD $4+$ lymphocytes $(26.15 \pm 5.6 \% ; \mathrm{p}<0.001)$, and increased absolute $(0.07 \pm 0.01 ; \mathrm{p}<0.01)$ and relative $(1.7$ $\left.\pm 0.9 \times 10^{9} / 1 ; \mathrm{p}<0.001\right)$ numbers of $\mathrm{NK}$ cells. Changes in humoral immune response were expressed by a significant increase in concentration of serum IgA $(1.14 \pm 0.51 \mathrm{~g} / \mathrm{l}), \mathrm{IgM}$ $(2.59 \pm 1.51 \mathrm{~g} / \mathrm{l}, \mathrm{IgG}(22.17 \pm 2.2 \mathrm{~g} / \mathrm{l})$, and circulating immune complexes by $2-4$ times in comparison with the healthy children. Study of serum concentrations of INF- $\gamma$, IL-4, IL-1, IL8 in children with HIV infection revealed a significant increase in INF- $\gamma$, and a decrease in IL-4, IL-1, IL-8 in comparison with healthy group.

Conclusions We revealed signs of the immune system impairment in children with perinatal HIV infection: clinical - manifestations of infectious and lymphoproliferative syndromes, and laboratory - combined immunodeficiency. HIV-infected children had an immune deviation towards Th2 response: increased IL-4 concentration and total IgE, and decreased INF- $\gamma$ in serum.

\section{GP205 MATERNAL PYREXIA AT BIRTH; A TRUE NEONATAL INFECTION OR NOT?}

Muhammad Abu Bakar, Aisha ljaz*, Aftab, Niazy Al-Assaf. University maternity hospital limerick, Limerick, Ireland

\subsection{6/archdischild-2019-epa.264}

Background Early onset neonatal sepsis(EOS) is defined as infection in the first 72 hours of life $^{1}$. It can cause serious morbidity and mortality therefore prophylactic antibiotics are required for high risk infants. ${ }^{1}$

Objective To determine the rate of positive blood cultures in babies treated in view of maternal pyrexia. To compare maternal high CRP and blood cultures with babies CRP and blood cultures.

Standard NICE guidelines Neonatal infection (early onset): antibiotics for prevention and treatment published: 22 August 2012. ${ }^{2}$

Methods Retrospective chart review was done on term babies admitted to NICU with maternal pyrexia form July 2016 to December 2016. Details of babies blood culture, CRP, and further workup e.g CSF studies and PCR studies were collected. Other details of maternal septic workup were also recorded. Data was compiled on Microsoft excel sheet and results analyzed.

Results Total 52 babies admitted from July 2016 to December 2016 in NICU with suspected EOS. 31\%(n=16) have other risk factors for EOS like maternal GBS, chorioamnionitis or PROM were excluded from the study. Only $3 \%(n=1)$ blood culture came back Micrococcus Luteus positive(contamination) with negative maternal culture. None of the mother involved in this study had positive blood culture. $28 \%(n=10)$ of the babies had CRP in the range of 6 to $30 \mathrm{mg} / \mathrm{L}$ while $3 \%(\mathrm{n}=1)$ of the babies had CRP above $50 \mathrm{mg} / \mathrm{L} .59 \%(\mathrm{n}=20)$ of the maternal CRP were less than $50 \mathrm{mg} / \mathrm{L}$. Only $6 \%(\mathrm{n}=2)$ of the mothers had CRP more than $200 \mathrm{mg} / \mathrm{L}$ compared to babies CRP less than $5 \mathrm{mg} / \mathrm{L}$.

Conclusion No correlation between baby CRP and maternal CRP in view of maternal pyrexia. None of the babies had positive blood culture in this cohort. Treating babies in view of maternal pyrexia alone as the risk factor for EOS should be reviewed to restrict unnecessary antibiotics use.

\section{REFERENCES}

1. Emily J. Weston, MPH, Tracy Pondo, MSPH, Melissa M. Lewis, MPH, Pat MartellCleary, MSW: The Burden of Invasive Early-Onset Neonatal Sepsis in the United States 2005-2008 Pediatr Infect Dis J. 2011 Nov; 30(11): 937-941

2. NICE guidelines: Neonatal infection(early onset): antibiotics for prevention and treatment(CG149); August 2012 